Ann. Biol. anim. Bioch. Biophys., I973, 13 (I), 93-105.

\title{
INFLUENCE DE LA NATURE DES MATIÈRES GRASSES INCORPORÉES DANS L'ALIMENT D'ALLAITEMENT SUR LE MÉTABOLISME LIPIDIQUE DE L'AGNEAU DE BOUCHERIE
}

\author{
B. AUROUSSEAU, M. THÉRIEZ* et Maryvonne DANIEL* \\ Station d'Études des Métabolismes, \\ * Station de Recherches sur l'Élevage des Ruminants, \\ Centre de Recherches de Clermont-Ferrand, I. N. R. A., \\ Theix Saint Genès Champanelle \\ 63110 Beaumont
}

\section{RÉSUMÉ}

La composition des dépôts adipeux superficiels d'agneaux élevés par leur mère (lot témoin) ou avec des aliments d'allaitement contenant de l'huile de coprah, de l'huile de palme ou du suif a été déterminée au sevrage (45 j), puis à go et 120 ou 140 jours (abattage.)

La composition des matières grasses ingérées avant sevrage modifie celle des dépôts adipeux superficiels au sevrage; des différences importantes subsistent jusqu'à l'abattage.

A l'abattage, la teneur en azote du muscle supra spinatus est identique pour les quatre lots mais sa teneur en matière sèche, en lipides et en énergie varie selon la nature des matières grasses incorporées aux aliments d'allaitement.

La tenue des dépôts adipeux de couverture de la carcasse varie selon le régime ; elle est reliée à leur teneur en acides gras saturés et à leur teneur en eau.

\section{INTRODUCTION}

Il est classiquement admis que les particularités de la digestion des lipides alimentaires chez le ruminant entraînent une grande stabilité dans la composition de ses dépôts adipeux (Tove, I960; GAR'TON et DUCAN, I969). Pourtant la constitution ou la présentation de la ration peut modifier la composition des tissus adipeux chez l'adulte (bovins : SHAw et al., I960; ovins : Tove et Matrone, I962). De même, la qualité des dépôts adipeux de couverture d'agneaux du type "agneaux de roo jours " élevés avec des laits artificiels semble inférieure et leur composition chimique différente de celles d'animaux nourris sous la mère (WILson et al., I967; Mol_énat et al., $\mathrm{r} 97 \mathrm{I})$. 
Dans l'expérience que nous présentons, nous avons déterminé 1'influence de la nature des matières grasses incorporées dans des aliments d'allaitement sur la composition des dépôts adipeux de couverture de l'agneau au sevrage (45 jours) et sur 1'orientation du métabolisme lipidique au cours de la phase d'engraissement, jusqu'à l'abattage. Un lot d'agneaux élevés par leur mère a été utilisé en référence.

\section{MATÉRIEL ET MÉTHODES}

\section{A. - Aliments}

Nous avons distribué 3 aliments d'allaitement dont la composition et la digestibilité figurent sur le tableau I. Ces aliments contenaient soit du suif qui est la matière grasse la plus fréquemment utilisée, soit de l'huile de coprah riche en acides gras à chaîne moyenne qui sont davantage métabolisés et déposés en proportions moindres que les acides gras longs (Bollinger, I963; Flanzy et al., 1970), soit de l'huile de palme riche en acide palmitique dont le métabolisme présente des particularités apparemment favorables à la croissance (MoHRHAUER et HolmaN, 1957). Ces trois matières grasses étaient apportées au taux de 23 ou $25 \mathrm{p}$. Ioo de la matière sèche pour obtenir des aliments isoénergétiques sur la base de l'énergie brute.

\section{TABIEAU I}

Composition et digestibilité des différents aliments d'allaitement

\begin{tabular}{|c|c|c|c|c|}
\hline \multirow{2}{*}{ Constituants (p. 100) } & \multicolumn{3}{|c|}{ Aliments d'allaitement } & \multirow{2}{*}{$\begin{array}{c}\text { Lait de brebis } \\
\text { Limousine } \\
\left(4^{\mathrm{e}} \text { et } 5^{\mathrm{e}} \mathrm{s}\right)\end{array}$} \\
\hline & Suif & Palme & Coprah & \\
\hline Poudre de lait écremé $\ldots . \ldots$. & 70,5 & 70,5 & 70,5 & \\
\hline Suif $\ldots \ldots \ldots \ldots \ldots \ldots$ & 23,0 & - & $\longrightarrow$ & \\
\hline Huile de palme $\ldots \ldots \ldots \ldots \ldots$ & $\underline{20,0}$ & 23,0 & - & \\
\hline Huile de coprah $\ldots \ldots \ldots \ldots$ & - & - & 25,0 & \\
\hline Lécithine $\ldots \ldots \ldots \ldots \ldots$ & 1,0 & 1,0 & 1,0 & \\
\hline Ferments lactiques $\ldots \ldots \ldots \ldots$ & 2,5 & 2,5 & 2,5 & \\
\hline Amidon de maîs et complément & & & & \\
\hline vitaminique $\ldots \ldots \ldots \ldots \ldots$ & 3,0 & 3,0 & 1,0 & \\
\hline
\end{tabular}

Composition (p. 100 de la MS)

\begin{tabular}{|c|c|c|c|c|}
\hline Matière sèche . . . . . . . . . & 96,1 & 96,3 & 96,6 & 17,6 \\
\hline Matières grasses .......... & 23,1 & 24,2 & 25,5 & 35,0 \\
\hline Matières azotées . . . . . . . . . & 24,9 & 23,7 & 24,2 & 27,0 \\
\hline Energie (kcal/g MS) . . . . . . . & 5,345 & 5,337 & 5,362 & 6,950 \\
\hline
\end{tabular}

Digestibilité (p. 100)*

\begin{tabular}{|c|c|c|c|c|}
\hline Matière sèche ........... & $95,7 \pm 0,6$ & $95,7 \pm 0,8$ & $97,0 \pm 0,6$ & \\
\hline Matières grasses $\ldots \ldots \ldots \ldots$ & $91,1 \pm 2,0$ & $92,2 \pm 0,2$ & $96,4 \pm 0,9$ & \\
\hline Matières azotées ........... & $95,5 \pm 0,0$ & $94,9 \pm 0,8$ & $96,0 \pm 0,4$ & \\
\hline Énergie $\ldots \ldots \ldots \ldots \ldots$ & $94,7 \pm 0,9$ & $95,0 \pm 0,8$ & $97,0 \pm 0,6$ & \\
\hline
\end{tabular}

* Mesures sur 4 agneaux par régime, pendant 7 jours du $7^{\mathrm{e}}$ au $15^{\mathrm{e}}$ jour après la naissance. 
Les agneaux disposaient à volonté du lait de remplacement qui était offert chaque jour à la température ambiante (THériez et al., I973). L'âge moyen au sevrage était de 45 jours.

Après sevrage, nous avons engraissé les animaux avec un aliment aggloméré complet composé de $48 \mathrm{p}$. Ioo de luzerne déshydratée, Io $\mathrm{p}$. Ioo de tourteau d'arachide, $20 \mathrm{p}$. Ioo d'orge, $20 \mathrm{p}$. Ioo de blé et $2 \mathrm{p}$. Ioo de complément minéral. Cet aliment qui avait des teneurs de $\mathrm{I} 5,4 \mathrm{p}$. IOo en matières azotées totales, I 2 p. Ioo en cellulose brute et $I, 8 \mathrm{p}$. Ioo en matières grasses, était offert chaque jour à volonté avec de la paille d'orge et de l'eau.

\section{B. - Animaux}

Nous avons utilisé au cours de cette expérience 55 agneaux mâles Limousins ou Romanov $\times$ Limousins. Séparés de leur mère 6 à $\mathrm{I} 2$ heures après la naissance, 45 d'entre eux ont été répartis, à l'âge de 3 jours, entre les 3 régimes expérimentaux, selon 3 lots homologues de I5 animaux élevés en groupes de 5.

Deux lots supplémentaires ont été constitués à 35 jours avec Io animaux élevés par leur mère. Ces agneaux, qui constituent les témoins, ont été sevrés au même âge et engraissés avec le même aliment granulé complet que les animaux des lots expérimentaux.

Les animaux ont été abattus soit lorsqu'ils ont atteint un poids vif de $35 \mathrm{~kg}$, soit à l'âge de $I_{4}$ o jours pour ceux qui n'avaient pas atteint ce poids.

\section{C. - Mesures}

Outre les mesures de qua ntités d'aliments ingérées, de gain de poids vif qui sont présenté par ailleurs (ThéRIEZ et al., I973), nous avons procédé aux mesures suivantes :

- Au sevrage (45 jours), puis à 90 jours, nous avons prélevé par biopsie un échantillon de gras de couverture à la base de la queue sur les 4 agneaux les plus lourds de chaque régime. A l'abattage, un nouvel échantillon de gras de couverture a été prélevé sur toutes les carcasses au même site qui a été choisi en raison des facilités de prélèvement sur l'animal vivant et de l'importance de ce dépôt sur la carcasse. Les échantillons prélevés à l'abattage ont été regroupés selon la vitesse de croissance c'est-à-dire l'âge des animaux (I 20 ou I 40 jours).

- Lors de l'abattage, l'état d'engraissement des carcasses a été déterminé par pesée du gras périrénal, du péritoine (toilette) et mesure de l'épaisseur du gras dorsal au niveau de la première vertèbre lombaire. La qualité des dépôts adipeux de couverture a été estimée et notée (de 3 pour un gras huileux à $\mathbf{5}$ pour un gras très ferme).

- Nous avons enfin prélevé sur 3 carcasses homologues, de chacun des quatre lots, le muscle sus-épineux (supra spinatus). Une bonne corrélation relie en effet la teneur de ce muscle en matière sèche, énergie et azote à celle de la masse musculaire totale de l'agneau (PrUD'HON, et al., 1972).

La teneur en eau des dépôts adipeux de couverture a été déterminée sur les échantillons regroupés selon l'âge et le régime par entraînement au benzène; leurs lipides ont été extraits après hydrolyse acide à chaud selon une méthode utilisée par Toullec et al. (1968) ; leur composition en acides gras a été déterminée par chromatographie en phase gazeuse de leurs esters méthyliques (détecteur à ionisation de flamme).

\section{RÉSULTATS}

Les rést'ltats zootechniques (vitesses de croissance des animaux et indices de consommation), publiés antérieurement ('THÉRIEZ et al., r973), sont résumés sur le tableau 2.

La nature de la matière grasse incorporée dans les aliments d'allaitement a modifié l'état d'engraissement des carcasses et la teneur de leurs dépôts adipeux de couverture. Les agneaux des lots " maternel » et "coprah » ont donné les carcasses les 
plus grasses (tabl. 2). L'épaisseur du gras dorsal est plus importante (différence non significative) et le gras périrénal significativement plus lourd dans les carcasses chez ces animaux que dans celles des agneaux des lots " sujf » et " palme ". Cependant les effets du régime sont en partie masqués par ceux de la vitesse de croissance. En effet, pour un même régime, les agneaux les plus âgés à l'abattage ont les dépôts adipeux de couverture les plus importants et, sauf dans le cas du lot "coprah" les dépôts périrénaux les moins lourds.

TABLEAU 2

Principaux résultats d'élevage

(Thériez et al., 1973)

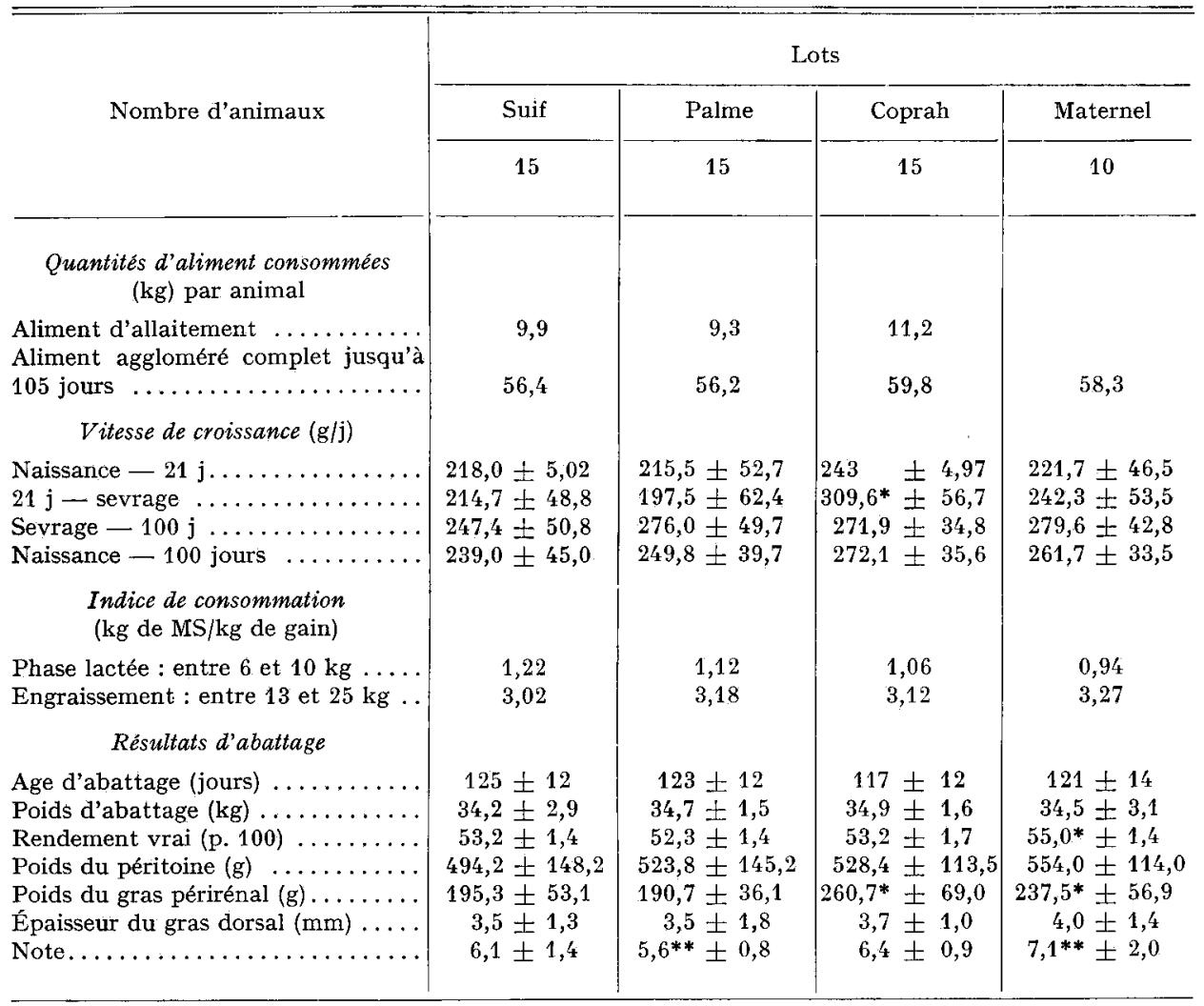

* Significativement différent $(P<0,05)$ des autres valeurs.

** Les 2 valeurs sont significativement différentes entre elles $(\mathrm{P}<0,05)$.

$\mathrm{Si}$ les teneurs en azote rapportées au gramme de muscle frais (supra spinatus) sont identiques d'un lot à l'autre (29,4 mg d'azote/g de muscle frais), les teneurs en matière sèche et en lipides varient, par contre, avec le régime. Les agneaux du lot " maternel " avaient les tissus musculaires les plus riches en matière sèche, en lipides 
et en énergie (tabl. 5) et les teneurs les plus faibles ont été observées sur les muscles des agneaux du lot " coprah ".

La composition en acides gras des dépôts constitués au cours de la phase lactée et prélevés par biopsie à 45 jours est fortement influencée par la nature des matières grasses de l'aliment d'allaitement (tabl. 3 et 4 ). Ainsi les acides gras à chaîne moyenne $\left(\mathrm{C}_{12}\right.$ et $\left.\mathrm{C}_{14}\right)$ ou les acides gras longs saturés $\left(\mathrm{C}_{16}\right.$ et $\mathrm{C}_{18}$ ) se retrouvent dans ces dépôts en proportion d'autant plus importante qu'ils sont plus abondants dans les aliments d'allaitement. Cependant, les agneaux du lot "coprah "font exception. Chez ces derniers 1'acide palmitique $\left(\mathrm{C}_{16: 0}\right)$ est plus abondant dans les dépôts que dans 1'aliment d'allaitement (fig. I).
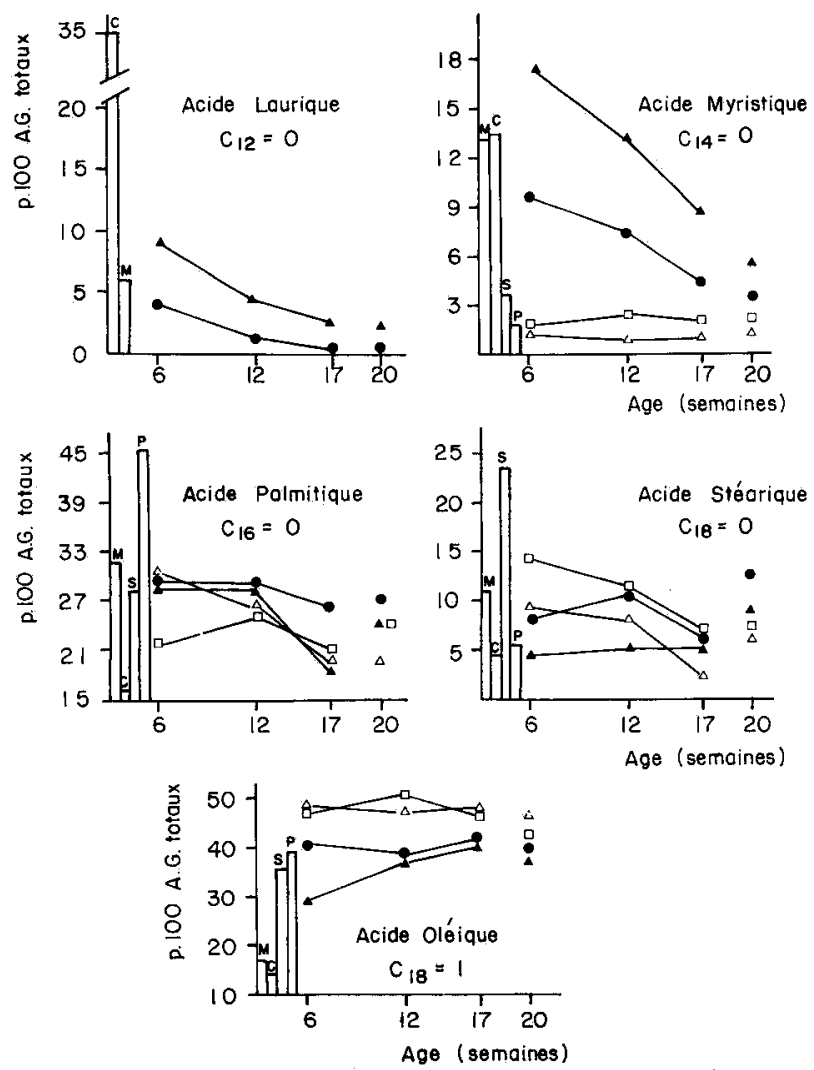

FıG. I. - Evolution en fonction de l'âge de la teneur en acides gras des dépóts adipeux superficiels de l'agneau selon le régime au cours de la phase lactée

0 Composition de l'aliment d'allaitement

- Lot " Maternel "

4 Lot "Coprah"

ㄴ Lot "Suif "

$\triangle$ Lot "Palme"

Les teneurs en acides gras mono-insaturés varient, par contre, indépendamment des apports. Les dépôts adipeux des agneaux des lots " maternel " et "coprah " sont plus riches en acide palmitoléique $\left(C_{18: 1}\right)$ que ceux des animaux des lots " suif " et " palme ". Chez les premiers, les dépôts contiennent, par contre, 2 à 2,4 fois plus d'acide 
oléique que les aliments d'allaitement alors que chez les seconds, ce rapport varie entre $I, 2$ et $I, 3$.

A l'âge de 90 jours (seconde biopsie), soit 45 jours après sevrage, selon la nature des matières grasses ingérées au cours de la phase lactée, les différences de composition des dépôts adipeux sont encore importantes (tabl. 3 et 4). La teneur des dépôts en acides gras longs saturés a augmenté sauf dans le cas des agneaux qui ont ingéré de l'huile de palme chez lesquels on trouve au contraire, un peu moins de ces acides. Les variations de teneur des dépôts en acides gras saturés totaux les plus fortes sont observées chez les animaux du lot "coprah " : les teneurs en acides gras à chaîne moyenne ont diminué et la teneur en acide oléique a augmenté.

\section{TABLEAU 3}

Composition des aliments d'allaitement

et des dépôts sous-cutanés des animaux au sevrage, à 90 jours et à l'abattage (1) Teneur en acides gras dont la chaîne comporte moins de 16 atomes de carbone $\left(^{2}\right)$

\begin{tabular}{|c|c|c|c|c|c|}
\hline & $\mathrm{C}_{8}$ & $c_{10}$ & $\mathrm{c}_{12}$ & $\mathrm{c}_{14}$ & $C_{15}$ \\
\hline Lait " Maternel " .......... & 2,9 & 11,5 & 6,6 & 13,2 & 0,3 \\
\hline $\begin{array}{r}\text { Tissu adipeux } \text { sous-cutané } \\
\text { à } 45 \text { jours } \ldots \ldots \ldots \ldots \ldots \\
\text { à } 90 \text { jours } \ldots \ldots \ldots \ldots \ldots \\
119 \text { jours } \ldots \ldots \ldots \ldots \\
140 \text { jours } \ldots \ldots \ldots \ldots\end{array}$ & $\begin{array}{l}- \\
-\end{array}$ & $\begin{array}{l}\overline{0,2} \\
0,2 \\
0,2\end{array}$ & $\begin{array}{l}1,9 \\
1,0 \\
0,2 \\
0,2\end{array}$ & $\begin{array}{l}9,9 \\
7,7 \\
4,8 \\
3,6\end{array}$ & $\begin{array}{l}-- \\
1,7 \\
2,9 \\
2,2\end{array}$ \\
\hline 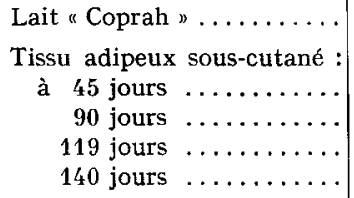 & $\begin{array}{l}- \\
- \\
-\end{array}$ & $\begin{array}{l}- \\
0,2 \\
0,1 \\
0,1\end{array}$ & $\begin{array}{l}9,1 \\
4,6 \\
2,8 \\
2,2\end{array}$ & $\begin{array}{r}17,3 \\
13,3 \\
8,8 \\
5,7\end{array}$ & $\begin{array}{l}- \\
0,8 \\
1,8\end{array}$ \\
\hline $\begin{array}{r}\text { Lait " Suif } " \ldots \ldots \ldots \ldots \\
\text { Tissu adipeux sous-cutané } \\
\text { à } 45 \text { jours } \ldots \ldots \ldots \ldots \\
90 \text { jours } \ldots \ldots \ldots \\
119 \text { jours } \ldots \ldots \ldots \ldots \\
140 \text { jours } \ldots \ldots \ldots \ldots\end{array}$ & $\begin{array}{l}- \\
- \\
-\end{array}$ & $\begin{array}{l}- \\
\overline{0,1} \\
0,1\end{array}$ & $\begin{array}{l}0,3 \\
0,6 \\
\frac{-}{0,3}\end{array}$ & $\begin{array}{l}2,0 \\
2,6 \\
2,1 \\
2,3\end{array}$ & $\begin{array}{l}- \\
\overline{1,2} \\
2,8\end{array}$ \\
\hline 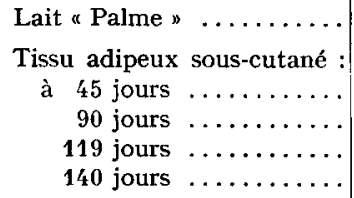 & $\begin{array}{l}- \\
- \\
-\end{array}$ & $\begin{array}{l}\overline{-} \\
0,1 \\
0,2\end{array}$ & $\begin{array}{l}0,4 \\
0,3 \\
0,1 \\
0,2\end{array}$ & $\begin{array}{l}1,7 \\
1,4 \\
1,5 \\
1,7\end{array}$ & $\begin{array}{l}- \\
1,1 \\
2,4 \\
2,9\end{array}$ \\
\hline
\end{tabular}

(1) Les analyses des dépôts à 45 et 90 jours ont été effectuées sur les animaux à croissance rapide abattus à 119 jours.

(2) A l'exception de quelques isomeres ou de quelques acides insaturés (0 à 3 p. 100 en tout) mal séparés. 


\begin{tabular}{|c|c|c|c|c|c|c|c|c|}
\hline 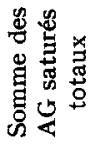 & $\bar{\infty}$ & 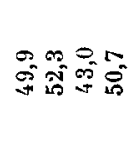 & $\tilde{o}_{\infty}^{\infty}$ & 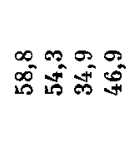 & $\begin{array}{l}\infty \\
i^{\circ}\end{array}$ & 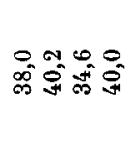 & है & 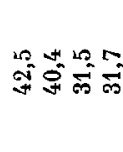 \\
\hline 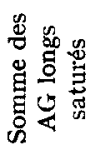 & $\hat{\tilde{a}}$ & $\overrightarrow{0}=0 \begin{array}{l}0 \\
0\end{array}$ & $\frac{\infty}{n}$ & 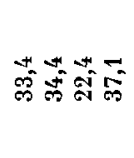 & $\frac{0}{i n}$ & 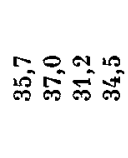 & $\frac{1}{n}$ & 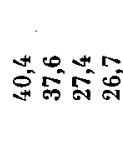 \\
\hline$\frac{\pi}{\ddot{0}}$ & $\approx$ & $\stackrel{12}{\theta}=\stackrel{0}{=}=$ & $\overrightarrow{0}$ & $\overrightarrow{0}=$ & $\stackrel{20}{0}$ & $\stackrel{9}{\because} \stackrel{0}{\rightarrow}$ & 1 & $1 \stackrel{0}{-1}=$ \\
\hline$\stackrel{\infty}{\ddot{\infty}}$ & $\stackrel{9}{=}$ & $\stackrel{\infty}{=} \stackrel{N}{=} \stackrel{\infty}{=} \stackrel{\infty}{=}$ & $\stackrel{9}{\xi}$ & $\begin{array}{l}\overrightarrow{0} \\
\sin \infty\end{array}$ & $\stackrel{\infty}{\infty}$ & 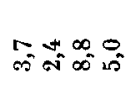 & $\stackrel{2}{5}$ & $\begin{array}{lll}4 & 40 & 0 \\
0 & 0 & 0\end{array}$ \\
\hline$\stackrel{\ddot{\omega}}{\tilde{S}}$ & $\stackrel{2}{5}$ & 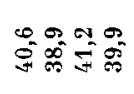 & $\vec{J}$ & 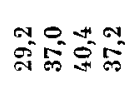 & N & 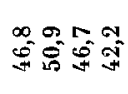 & 占 & 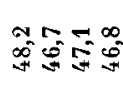 \\
\hline$\stackrel{\infty}{\xi}$ & $\hat{g}$ & مَ & 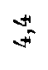 & 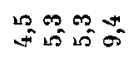 & $\stackrel{4}{a}$ & 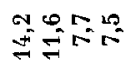 & $\infty$ & 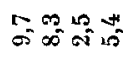 \\
\hline$\ddot{ت}$ & 1 & $1 \stackrel{\infty}{\approx}=\infty$ & 1 & $1 \stackrel{0=0}{9}$ & 1 & $11=0$ & 1 & $1 \stackrel{m}{\rightarrow} \rightarrow \infty$ \\
\hline$E_{j}$ & 1 & $\mid \stackrel{\infty}{\infty} \stackrel{0}{-}=$ & 1 & 1110 & I & 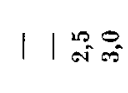 & | & $1 \begin{array}{ll}102 & 0 \\
\text { of } & 5\end{array}$ \\
\hline
\end{tabular}


Lors de l'abattage nous avons distingué deux catégories d'animaux, les uns à croissance rapide (abattage à I2o jours environ) qui correspondent aux agneaux sur lesquels ont été pratiquées les biopsies, les autres à croissance plus lente (abattage à I40 jours). Quelle que soit la vitesse de croissance, les teneurs en acides gras saturés totaux des dépôts adipeux sont plus faibles à l'abattage qu'à 45 ou go j. Lies agneaux à croissance rapide ont les dépôts les moins riches en acides gras de ce type (tabl. 3 et 4). Chez ces animaux, la teneur en acides gras longs saturés a diminué plus fortement entre 45 jours et l'abattage que chez les agneaux abattus à r40 jours. Les valeurs obtenues alors sont inférieures de 3 à I 5 points à celles observées chez les agneaux à croissance plus lente sauf dans le cas du lot " palme " où l'écart est inversé et très faible $(0,7$ points).

Cette évolution de la teneur en acides gras longs saturés a, en outre, été différente selon la nature des matières grasses ingérées au cours de la phase lactée. Chez les animaux abattus à r2o jours, les plus fortes diminutions ont été observées dans les lots " coprah " et " palme " (respectivement — II et - I3 points entre sevrage et abattage contre $-3,4$ et $-4,5$ pour les lots " maternel " et " suif »). A l'exception du cas des agneaux du lot "palme" (- I3,7 points), des différences moins marquées (- I,2 points pour le lot " suif ") ou inverses (respectivement $+6,4$ et $+3,7$ points pour les lots " maternel " et " coprah ") ont été observées entre $45 \mathrm{j}$ et l'abattage, chez les animaux à croissance plus lente. Ces variations sont imputables pour leur plus grande part aux variations des teneurs en acide stéarique.

\section{TABLEAU 5}

Caractéristiques du muscle sus-épineux (supra-spinatus) des animaux abattus à 120 jours

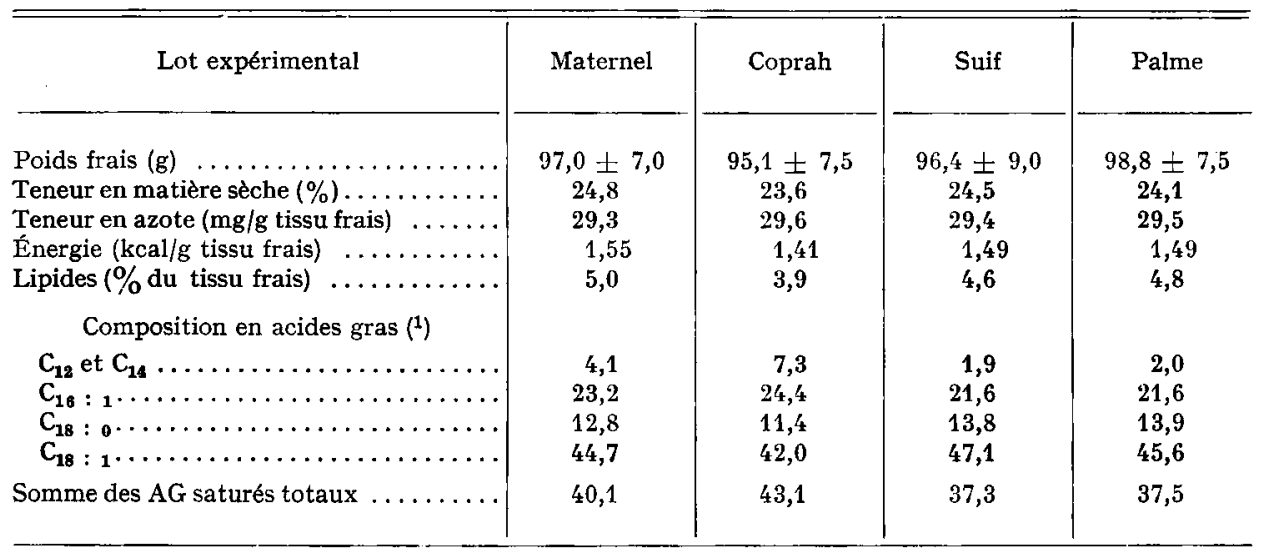

(1) Principaux AG seulement.

L'analyse des lipides intramusculaires (supra spinatus) des agneaux abattus à I20 jours confirme les résultats précédents (tabl. 5). Comme dans le cas des dépôts adipeux externes, ce sont les lipides intramusculaires des agneaux des lots " suif " et " palme " qui ont les plus fortes teneurs en acide oléique $(47, \mathrm{I}$ et $45,6 \mathrm{p}$. Ioo contre 
44,7 et 42,0 p. roo pour les animaux des lots " maternel " et "coprah ") et qui sont les plus pauvres en acides gras saturés totaux (respectivement 42,2 et 43,0 p. Ioo contre 46,3 et 49 ,I p. roo).

La vitesse de croissance et la nature des matières grasses ingérées au cours de 1a phase lactée ont également un effet sur la tenue des dépôts adipeux externes, bien que l'écart moyen entre l'âge d'abattage des agneaux des différents régimes ne soit que de 7 jours. Dans le cas des lots " maternel " et " coprah », les agneaux abattus les plus âgés ont les dépôts les plus fermes alors qu'on peut observer l'inverse pour les 2 autres lots. La moyenne des notes attribuées diminue du lot " maternel " au lot " coprah " puis aux lots " suif » et " palme " (tabl. 2). La différence"entre les lots " maternel " et " palme " est significative.

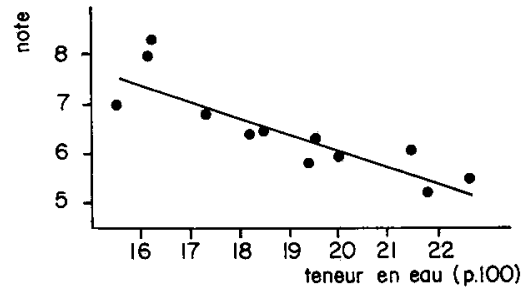

(อ)

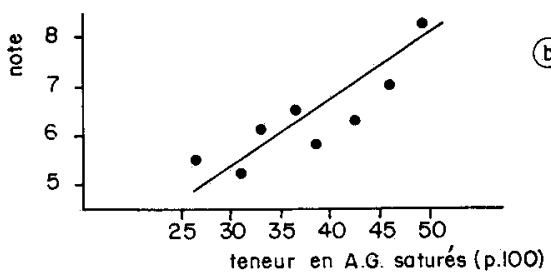

(b)

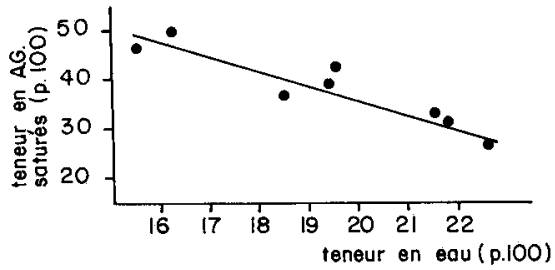

(c)

Fig. 2. - Relation entre la temue des dépôts adipeux, leur teneur en eau et leur teneur en acides gras saturés

a) Liaison tenue des dépôts - teneur en eau $r=-0,72(\mathrm{P}<0,05)$;

b) Liaison tenue des dépôts - teneur en acides gras saturés $r=+0,80(\mathrm{P}<0,05)$

c) Liaison teneur en acides gras saturés - teneur en eau $r=-0,95(\mathrm{P}<0,05)$

La tenue des dépôts adipeux est liée de manière significative à leurs teneurs en eau $(r=-0,72$, fig. $2 a)$ et en acides gras saturés $(r=+0,80$, fig. $2 b)$, et il existe une liaison plus étroite encore entre les teneurs des dépôts adipeux en eau et en acides gras saturés $(r=-0,95$ fig. $2 c)$. Les dépôts adipeux de couverture les plus fermes sont ceux dont la teneur en eau est la plus faible et la teneur en acides gras saturés la plus élevée. 


\section{DISCUSSION ET CONCLUSION}

La composition des matières grasses incorporées dans les aliments d'allaitement que nous avons utilisés correspond aux valeurs moyennes indiquées par HILDITCH et WILIIAMS (I964) ou WoLFF (I968) sauf dans le cas de l'aliment coprah. Les teneurs en acides laurique et myristique du coprah utilisé sont anormalement faibles (respectivement 35,3 et 13,5 p. Ioo, 45 et I7 p. Ioo selon WoL,FF) et sa teneur en acide linoléique trop élevée 4,6 au lieu de I p. Ioo en moyenne. Si 1'origine de ces écarts n'est pas connue, (mélange éventuel de matières grasses lors de la fabrication du lait), les différences importantes de composition des aliments d'allaitement ont cependant permis de mettre en évidence l'influence des matières grasses alimentaires sur la composition des dépôts adipeux externes de 1'agneau préruminant (biopsie à 45 jours). Ce résultat est en accord avec ceux de STOKES et WAI,KER (I970) sur l'Agneau, et ceux de Toullec et Mathieu (I969) sur le Veau; il montre que la composition des dépôts adipeux du jeune préruminant dépend de celle des matières grasses qu'il ingère, comme chez le monograstrique.

Des écarts importants subsistent à l'abattage entre les proportions d'acides gras à chaîne moyenne ou longue dans les dépôts adipeux externes des animaux des différents lots. Ces écarts ne peuvent être dus aux différences de quantités d'acides gras ingérées après le sevrage. Au cours de la phase d'engraissement, les agneaux qui ont consommé des quantités très voisines d'un même aliment aggloméré complet (tab1. 2), ont en effet ingéré des quantités de matières grasses sensiblement identiques, comprises entre I,I et I,2 kg soit deux fois moins qu'au cours de la phase lactée (respectivement $2,25,2,28$ et $2,85 \mathrm{~kg}$ pour les lots " palme ", " suif » et " coprah ").

De plus, l'influence des acides gras déposés pendant la période d'allaitement ne peut expliquer les écarts observés. On sait, en effet, (BÉNÉVENT, I97I) que l'agneau mâle dont la croissance demeure régulière, subit entre ro et $I_{5} \mathrm{~kg}$ de poids vif, une fonte importante du tissu adipeux. En outre, les dépôts adipeux constitués après le sevrage, à partir des matières grasses alimentaires ou des synthèses endogènes qui constituent la principale origine des dépôts adipeux externes chez le mouton (GARTON et DUNCAN, I969), sont 6 à 8 fois plus importants que ceux déposés au cours de la phase lactée. Or, la teneur en acide myristique des dépôts adipeux externes des agneaux des lots " coprah " ou " maternel " n'est réduite que de moitié environ. Dans le même temps, la teneur en cet acide des dépôts adipeux externes des agneaux des deux autres lots reste constante quel que soit 1'âge des animaux.

Par ailleurs, les écarts observés au moment de l'abattage en ce qui concerne les proportions des autres acides gras dans les dépôts adipeux externes des agneaux ne peuvent pas s'expliquer par une simple dilution des graisses corporelles constituées avant le sevrage. Ainsi, la teneur en acide palmitique des dépôts adipeux externes des agneaux élevés par leur mère décroît faiblement au cours de la phase d'engraissement pour atteindre une valeur (27 p. Ioo) voisine de celle observée par HiLdirch et Wiliriams (I964) chez l'adulte. Dans le cas des autres lots, on observe, au moment de l'abattage, des valeurs anormalement faibles de la teneur des mêmes dépôts en cet acide : respectivement I9 à 24,2 I à 24 et I9 à 20 p. Ioo pour les agneaux des lots 
" coprah ", "suif " et " palme ". Or, au moment du sevrage, la teneur de ces dépôts en acide palmitique était très voisine dans le cas des agneaux des lots " maternel ", " coprah " et " palme " (tabl. 4).

Les différences observées ont donc pour origine une modification du métabolisme du tissu adipeux chez les animaux expérimentaux. Par analogie avec les résultats obtenus sur rat (BolLINGER, I963, KRITChEvsky et al., I967, KAUNITz et JoHnson, I968, Aurousseau et al., r970-72), l'accroissement de la teneur des dépôts des agneaux des lots " maternel " et " coprah " en acides gras longs saturés, entre le sevrage et I40 jours, pourrait ainsi s'expliquer, par un effet favorable des acides gras à chaîne moyenne. Ceux-ci favoriseraient l'enrichissement des dépôts adipeux externes en acides gras longs saturés pendant la phase d'alimentation lactée et cet effet subsisterait après le sevrage. De même, les apports exogènes d'acide palmitique inhibent chez le monogastrique la synthèse de cet acide (BORTz et LYNEN, I963 ; BolLINGER, I963). La réduction de la teneur des dépôts des agneaux du lot "palme " en cet acide pendant la phase d'engraissement pourrait s'expliquer, par la permanence d'une telle inhibition induite au cours de la phase d'alimentation lactée, les fortes teneurs en acide palmitique des dépôts de ces animaux au moment du sevrage étant d'origine exogène.

Il serait intéressant de connaitre les causes de la permanence des orientations du métabolisme lipidique induites au cours de la phase d'alimentation lactée. Dans l'état actuel de nos connaissances, nous en sommes réduits à émettre des hypothèses. L'enrichissement en acides gras insaturés des dépôts adipeux des animaux abattus à I20 jours semble pouvoir s'expliquer, en effet, par une succession de phases d'anabolisme accru (GELLHORN et BENJAMIN, I965), et de remobilisations (DI GroRGIO et al., I962), dues au niveau d'ingestion élevé de ces animaux. L'écart plus marqué chez les agneaux des lots " maternel " et surtout " coprah " entre la teneur en acides gras insaturés des dépôts sous-cutanés dos animaux selon l'âge d'abattage (I20 ou I40 j) pourrait alors provenir de différences d'activité des tissus adipeux concernés d'un lot à l'autre. Or, la taille des cellules d'un tissu adipeux est le facteur principal de l'activité de ce tissu (LAvau, r970; Shapiro, I97I) et on sait, de plus, que les modifications de cellularité (mise en place de cellules plus petites et plus nombreuses) sont le facteur principal des effets irréversibles de la nutrition du nouveau-né sur la croissance ultérieure (WINICK et NoBLE, I967; RoBINson, I97I). On peut donc penser à une mise en place de tissu adipeux de cellularité et d'activité variable en fonction de la nature des matières grasses ingérées pendant la phase d'alimentation lactée. Il convient, cependant, de noter que plusieurs facteurs semblent intervenir pour modifier le degré d'induction du métabolisme par la nature des acides gras de 1'aliment d'allaitement ou pour en diminuer les effets en fin de période d'engraissement. Ainsi, dans le cas d'agneaux qui reçoivent des aliments d'allaitement à base de lait écrémé et de suif, la quantité d'acides gras longs ingérés par les animaux ('THÉRIEZ et MoLÉNAT, I972) ainsi que la durée de l'intervalle sevrage-abattage (MolÉnA' et al., I97r) semblent aussi capables de modifier la composition des tissus adipeux et leur tenue.

La liaison que nous observons entre la fermeté des tissus adipeux de couverture et leur teneur en eau ou en acides gras saturés concorde avec des observations antérieures sur ovins (JACOBS et al., I972; THÉRIEZ et MOLÉNAT, I97I) et avec celles de Fi,ANZy et al. (I970) sur porcs. Pour DuCAN et al. (I972) et par GARTon et al. (I972), 
par contre, l'accroissement de la teneur des dépôts adipeux externes en acides gras ramifiés serait à l'origine de la diminution de leur fermeté. Il faut noter, toutefois, que, dans les conditions expérimentales adoptées par ces auteurs (aliment d'engraissement comporte $96 \mathrm{p}$. Ioo de céréales et tourteaux contre $50 \mathrm{p}$. Ioo dans notre expérience), la teneur des dépôts adipeux des animaux en acides gras à chaîne impaire ou en acides gras à chaîne ramifiée s'élèvent respectivement jusqu'à 7 et I2 p. Ioo, soit 2 à 4 fois plus que dans le cas de notre expérience.

Reçu pour publication en octobre 1972.

\section{SUMMARY}

\section{THE INFLUENCE OF THE TYPE OF FAT IN THE MILK SUBSTITU'TE ON LIPID METABOLISM IN FATTENING LAMBS}

The influence of the type of fats included in the milk substitute on lipid metabolism and composition of external fat depos was studied in young lambs from weaning at 45 days to slaughter at $\mathrm{I} 20$ or $\mathrm{I}_{4} \mathrm{O}$ days of age.

The three sources of fat were tallow, palm oil and coconut oil. These three fats were compared with each other and with ewe's milk (tables I and 2).

Fatty acid composition of external fat at weaning depended on that of the fat ingested (tables 3 and 4 , fig. I). During the fattening periode the animals which had ingested the different experimental diets had external fat with composition which tended to approach that in the control lambs. However, there were still large differences at slaughter (I 20 or I40 days). The lambs which had been given coconut oil had fat with composition close to that in the control lambs, reared by their mothers. The external fat of lambs which had been given tallow or palm oil had less lauric and myristic acids and more oleic acid (tables 3 and 4 , fig. 1 ).

The composition of the intramuscular fat of the supra spinatus muscle was close to that of the external fat. The nitrogen content of his muscle was the same in lambs of all groups, but dry matter, lipids and energy increased in the order coconut oil, tallow and palm oil, maternal milk.

The degree of fattening in carcasses varied with the nature of the fat included in the milk substitute. The carcasses were classed in the following order : maternal milk and coconut oil, tallow and palm oil (table 2). The quality of the adipose deposits varied according to the diet. The firmest fats, which had the highest grading, were in carcasses of the suckled lambs, followed in order by coconut oil, tallow and palm oil (table 2).

There was a significant correlation between the grading of the fat and its saturated fatty acid content $(r=+0.80)$ or its moisture content $(r=-0.72)$ (fig. 2 and 3$)$. The results are discussed and it is shown that there is an effect of the fat ingested before weaning on lipid metabolism in adipose tissue after weaning.

\section{RÉFÉRENCES BIBLIOGRAPHIQUES}

Aurousseau B., De Groot L., Bouvier J.-C., Vermorel M., I97o. Utilisation métabolique des acides gras courts, moyens et longs par le rat en croissance. In : "Energy metabolism of farm animals". Proc. 5th Symp. Vitzmau, Switzerland (éd. A. Schurch and C. WenK), I97o, 189.

Aurousseau B., De Groot L., I972. Influence de l'acide caprylique sur le métabolisme des autres acides gras chez le rat en croissance. Ann. Biol. anim. Bioch. Biophys. (sous presse).

BÉnt́vent M., Ig7r. Croissance relative pondérale postnatale chez les deux sexes des principaux tissus et organes de l'agneau Mérinos d'Arles. Ann. Biol. anim. Bioch. Biophys., 11, 5-39.

BOLIINGER J. N., I963. The metabolism of fatty acids derived from dietary Triglycerides. $\mathrm{Ph}$. D. Thesis Univ. Texas.

Bortz W. W., LYNen F., I963. Elevation of long chain acyl CoA derivatives in livers of fasted rats. Biochem. Ztschr., 339, 77-82. 
Di Giorgio J., Bonanno R. A., Hegsted D. M., 1962. Effect of diet upon the in vitro metabolism of rat epididymal adipose tissue. J. Nutr., 78, 384-39I.

Duncan W. R. M., Orskov E. R., Garton G. A., I972. Fatty acid composition of triglycerides of lambs fed on barley based diets. Proc. Nutr. Soc., 31, ig A.

Flanzy J., François A.-C., Rérat A., r970. Utilisation métabolique des acides gras chez le porc. Ann. Biol. anim. Bioch. Biophys., 10, 603-620.

Garton G. A., Duncan W. H. R., r969. Composition of adipose tissue triglycerides of neonatal and year-old lambs. J. Sci. Fd. A gri., 20, 39-42.

Garton G. A., Hovel F. D. de B., Duncan W. R. H., 1972. Effect of dietary propionate on the fatty acid composition of lamb triglycerides. Proc. Nutr. Soc., 31, 20 A.

Gellhorn A., Benjamin W., 1965. Fatty acid biosynthesis and RNA function in fasting aging and diabetes. Adv. enzym. Regul., 4, I9-26.

Hilditch T. P., Williams P. N., I964. The chemical constitution of natural fats. p. I04-II2. Chapman and Hall, London.

Jacobs J. A., Field R. A., Botkin M. P., Kaltenbach C. C., Riley M. L., I972. Effects of testosterone enanthate on lamb carcass composition and quality. J. Anim. Sci., 34, 30-36.

KaUnitz H., Johnson R. E., 1968. Nutritional properties of M.C.T. J. A. O. C. S., 45, I9-22.

Kritchevsky D., Shirley A., TEPPER, I967. Influence of M.C.T. on cholesterol metabolism in rats. J. Nutr., 86, 67-72.

Lavau M., I970. Le tissu adipeux. Cahiers Nutrition diététique, 5, 69-75.

Mohrhauer M., Holman R. T., 1967. Metabolism of linoleic acid in relation to dietary saturated fatty acids in the rat. J. Nutr., 91, 528-534.

Molénat G., Theriez M., Aguer D., I97I. L'allaitement artificiel des agneaux. I. Détermination de l'âge minimal au sevrage pour la production d'agneaux de boucherie. Ann. Zootech., 20, 339-420.

Prud'hon M., Reyne Y., Garambors Y., I971. Estimation de la composition corporelle d'agneaux Mérinos d'Arles abattus à des stades de croissance compris entre la naissance et un an. Ann. Zootech., 21, 209-309.

Robrnson D. W., r97r. Cellular basis for changes in body composition. J. Anim. Sci., 33, 4I6-420.

Shapiro B., I97I. Regulation of adipose tissue size. "La régulation du bilan énergétique chez l'Homme " C. R. I er Cong. Soc. Nutr. Diet., Paris (sous presse).

Shaw J. C., Ensor W. L., Tellechea M. F., LEe S. D., I96o. Relation of diet of rumen volatile fatty acids, digestibility, efficiency of gain and degree of unsaturation of body fat in steers. $J . N u t r$., $71,203-208$.

Srokes G., Walker D. M., I97o. The nutritive value of fat in the diet of the milk fed lamb. 2. - The effect of different dietary fats on the composition of body fats. Brit. J. Nutr., 24, 435-440.

Thériez M., Molénat G., I972. Influence de la nature et du taux d'incorporation de la matière grasse dans les aliments d'allaitement pour agneaux sur la vitesse de croissance et la qualité de la carcasse. Proc. II Internat, milkreplacer symp., 1972, p. 53-62, National renderers association. Brussels, Belgium.

Thériez M., Molénat G., Daniel, M., Aurousseau B., ig73. L'allaitement artificiel des agneaux. III. Influence de la nature de la matière grasse incorporée dans l'aliment d'allaitement Ann. Zootech. (sous presse).

Toullec R., Flanzy J., Rrgaud J., ig68. Dosage des lipides des fèces. Ann. Biol, anim. Bioch. Biophys., 8, 28I-289.

Touldec R., Mathied C.-M., I967. Utilisation digestive des matieres grasses et de leurs principaux acides gras par le veau préruminant à l'engrais. Influence sur la composition corporelle. Ann. Biol. anim. Bioch. Biophys., 9, I39-145.

Tove B. B., I960. The origin of depot fat. J. Dairy Sci., 43, I354-1360.

Tove S. B., Matrone G., I962. Effect of purified diets on the fatty acid composition of sheep tallow. J. Nutr., 76, 27 I-277.

Wilson P. N., Hugues L. D., Svensgaard C., Starke J. S., r967. Carcass quality studies with lambs : the effect of normal rearing, early weaning at 3 weeks and artificial rearing on fat quality. Proc. C. I. R. C. R. A., conf. Dublin, 88-89.

Winick M., Noble A., 1967. Cellular response with increased feeding in neonatal rats. J. Nutr., 91, I79-182.

Wolfr J. P., rg68. Manuel d'analyse des corps gras. p. 313 Éditions Azoulay, Paris. 